\title{
Gradhiva
}

GRADHIV

Revue d'anthropologie et d'histoire des arts

$21 \mid 2015$

Création plastique d'Haïti

\section{Par-delà le structuralisme : Maurice Godelier, lecteur matérialiste de Claude Lévi-Strauss}

Laurent Berger

\section{(2) OpenEdition}

1 Journals

Édition électronique

URL : http://journals.openedition.org/gradhiva/2995

DOI : 10.4000/gradhiva.2995

ISSN : 1760-849X

Éditeur

Musée du quai Branly Jacques Chirac

Édition imprimée

Date de publication : 1 février 2015

Pagination : 222-247

ISBN : 978-2-35744-075-3

ISSN : 0764-8928

Référence électronique

Laurent Berger, « Par-delà le structuralisme : Maurice Godelier, lecteur matérialiste de Claude Lévi-

Strauss », Gradhiva [En ligne], 21 | 2015, mis en ligne le 01 février 2015, consulté le 22 septembre 2020 URL : http://journals.openedition.org/gradhiva/2995; DOI : https://doi.org/10.4000/gradhiva.2995

(c) musée du quai Branly 


\section{Par-delà le structuralisme: Maurice Godelier, lecteur matérialiste de Claude Lévi-Strauss}

par Laurent Berger

Maurice Godelier, Lévi-Strauss. Paris, Seuil, 2013.

Depuis 1952, la liste est longue - au moins une parution chaque année - des ouvrages dédiés à l'œuvre de Claude Lévi-Strauss. Le dernier en date, publié par Maurice Godelier, constitue un de ses inventaires les plus méthodiques, exposant de façon didactique, texte après texte, année après année, la démarche comparative, la rigueur démonstrative et la portée théorique - souvent révolutionnaires - des quelque deux cents articles et vingt et un ouvrages que Lévi-Strauss a consacrés durant sa vie à l'épistémologie des sciences sociales, aux réflexions sur la condition humaine, ainsi qu'à l'étude de la parenté, de l'art, des mythes et des rites. De cette «torsade à cinq brins» ne sont déroulées et décodées que les séquences propres à la parenté et aux connaissances mythico-rituelles; mais cette remontée de l'Amazone en pirogue à double hélice, aux côtés des soleils structuralistes et des vieilles lunes marxistes, reste d'une clarté et d'une exhaustivité remarquables pour appréhender d'un seul tenant l'ensemble du chemin accompli. Ce bilan critique est en effet dressé à l'aune d'un double parcours, en aval et en amont, de l'œuvre de Lévi-Strauss: à l'embouchure, tout d'abord, de nouveaux faits empiriques et de nouvelles analyses postérieures à sa réalisation («les failles et les limites de son œuvre qui ne sont pas toujours de son fait, mais souvent l'effet de son temps", p. 263); à la source, ensuite, de ses divergences théoriques avec les positions défendues par Godelier au terme de sa propre odyssée anthropologique, tant sur la prohibition de l'inceste et la nature de la pensée humaine que sur les fondements et l'historicité des sociétés.

Cette double lecture est d'autant plus intéressante que ce dernier se réclame ouvertement des principes de l'analyse structurale communs à Karl Marx et Lévi-Strauss, tout en appliquant ces préceptes aux domaines de la vie sociale volontairement laissés dans l'ombre par son aîné (l'économie et la politique). Le premier principe, on le sait, est un postulat matérialiste: les rapports des hommes entre eux et avec les non-humains impliquent à la fois la mise en œuvre d'opérations cognitives propres aux "structures universelles de l'esprit humain» et le jeu des propriétés objectives de ces rapports dans l'espace et le temps, au-delà de la représentation que s'en font les êtres humains. Le deuxième est l'idée que ces rapports forment "système " (c'est-à-dire un ensemble organisé de termes et de relations), que chaque type de système se différencie par sa «structure " (au sens où la modification d'un élément ou d'une relation entraîne la reconfiguration de l'ensemble en variante possible d'un «groupe de transformations»), et que ces structures expliquent en dernière analyse la logique de leur fonctionnement. Le troisième principe, enfin, est le parti pris rationaliste de considérer une structure comme indirectement perceptible et toujours reconstruite a posteriori, ce qui suppose d'étudier ses propriétés 


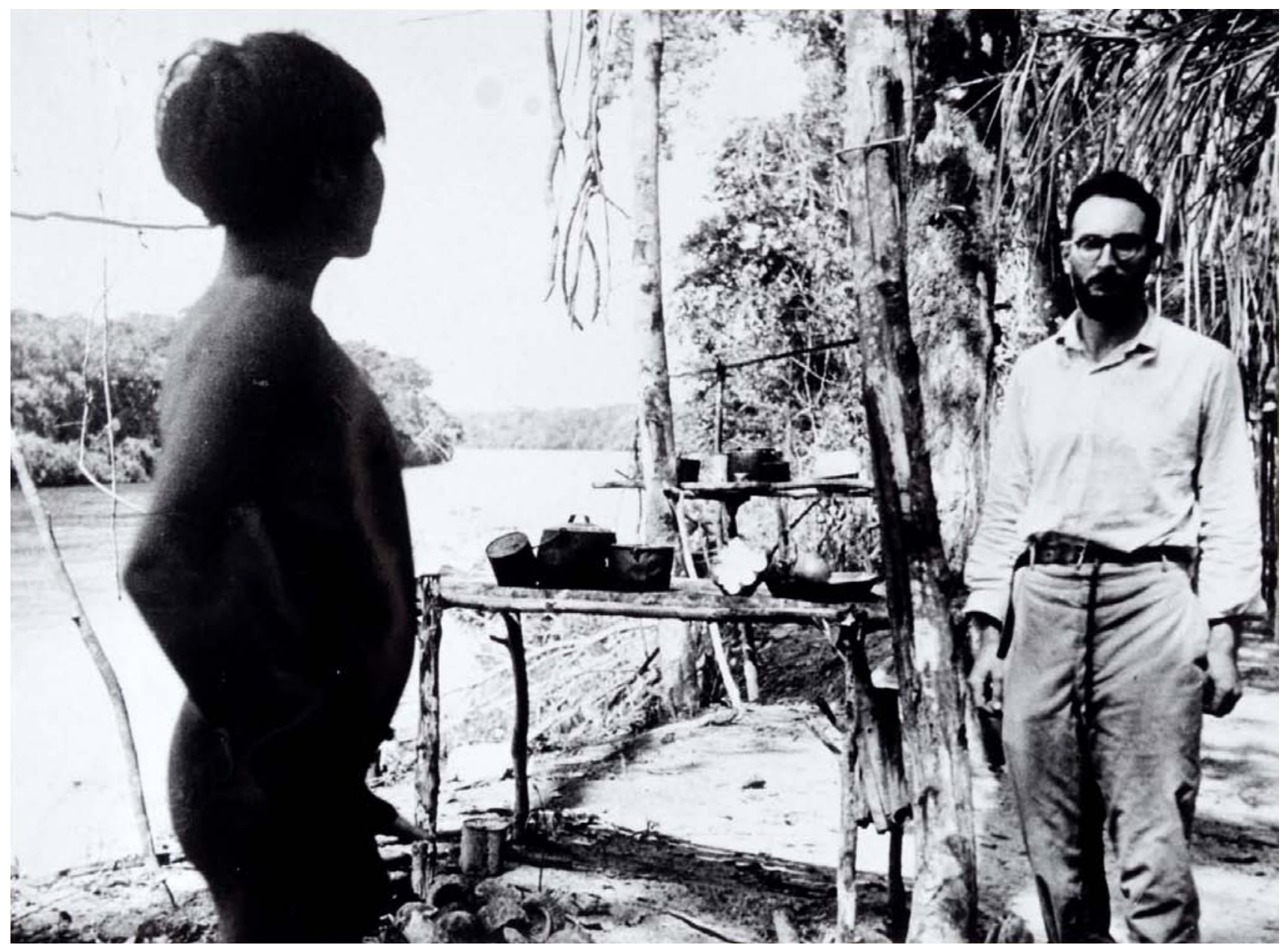

fig. 1

Lévi-Strauss et un Indien

tupi-kawahib au bord du

fleuve Machado, novembre

1938. () Archives CLS/Cl.

Patrick Léger-Gallimard.

Photo parue in Vincent

Debaene et Frédérick

Keck, Claude Lévi-Strauss,

L'homme au regard

éloigné, 2009, Collection

Découvertes Gallimard,

pp. 36-37. 
et ses variantes transformatives avant la genèse de ses composantes et l'historique de leurs assemblages (p. 30).

Godelier insiste à raison sur ce qui constitue dans l'analyse structurale un guide pratique pour inférer l'existence de tel et tel type de structure, et en même temps un garde-fou pour circonscrire étroitement leur diversité phénoménale au niveau des activités sociales observées: ce fil d'Ariane, c'est la correspondance logique posée entre les modèles abstraits de structure extraits de la vie sociale et ceux tirés des activités mentales (pour Lévi-Strauss, «l'ethnologie est d'abord une psychologie ${ }^{1}$ »).

II est donc question de structures propres aux phénomènes religieux et familiaux dans cette relecture de Lévi-Strauss par Godelier: la "formule canonique", l'«armature codée, schématique et sémantique des mythes", l'«atome de parenté», l'«échange différé, restreint et généralisé», les «alliances semi-complexes crow-omaha» ou les "maisons» sont ainsi systématiquement explicités et revisités. Mais il est aussi question des structures cognitives propres au cerveau qui rendent possible en filigrane l'expression de ces dernières, à l'instar de l'analogie proportionnelle $(A: B:: C: D)$, des schèmes, des procédures classificatoires, de la théorie de l'esprit, ou bien encore de la faculté d'imagination (explorée à travers l'enchaînement des déductions «empirique» et «transcendantale»). Cette approche méticuleuse, à la fois chronologique et thématique, révèle à quel point ces structures universelles de l'esprit humain ont toutes été mises en exergue par LéviStrauss, notamment dans son analyse du totémisme et de la mythologie amérindienne, bien avant le développement des recherches en sciences cognitives sur le sujet.

II ressort de ce parallélisme, omniprésent et propre à l'analyse structurale lévi-straussienne, trois défis majeurs adressés aux ethnologues en pirogue, soucieux, comme Godelier, de voyager dans la jungle des us et coutumes avec, à bord, un gouvernail «psychologue» et un propulseur «historien ». Le premier est de comprendre les systèmes fondés sur l'articulation de structures différentes, que ce soit à un niveau relativement simple (celui de la traduction d'une structure cognitive dans la modélisation d'une organisation sociale) ou bien à un degré de complexité plus élevé (celui de la compatibilité des structures entre elles). Le deuxième est de saisir les seuils au-delà desquels les transformations irréversibles ou l'articulation supplémentaire d'une ou plusieurs structures conduisent à l'émergence d'un système radicalement distinct des précédents. Le troisième est de circonscrire la place revenant aux événements et à l'agentivité humaine dans la reproduction ou l'évolution des systèmes dans le temps, et donc dans la transformation ou non de leurs structures. Ce n'est pas le moindre mérite de ce travail colossal que de s'attaquer à ce triple défi complémentaire, à travers un plaidoyer pour le rapprochement de l'histoire, de l'ethnologie et de la psychologie qui prolonge, et souvent réoriente, le tracé fluvial emprunté par Lévi-Strauss.

Du système fondé sur une combinaison structurelle Le système des attitudes parentales

L'atome de parenté est un bon exemple chez Lévi-Strauss de la
1. Ainsi, l'échange restreint, différé et généralisé présuppose trois «structures" de l'esprit humain: les quatre variantes de l'apprentissage instrumental (la notion de règle en tant que telle: faire/ ne pas faire pour obtenirl éviter de), le schème conceptuel de réciprocité et la faculté d'imagination attribuant aux protagonistes de l'échange une qualité non inhérente au transfert de valeur consenti (le caractère synthétique du don). 
traduction d'une structure cognitive dans l'organisation de relations sociales élémentaires. En l'occurrence, un raisonnement analogique délimite le «système des attitudes " des individus connectés par des termes de parenté, sur la base combinée de trois liens de parenté. Ces derniers sont, d'une part, les différentes formes de consanguinité et d'affinité (entre germains, parents et enfants ou ascendants et descendants d'un côté; entre conjoints ou beaux-parents de l'autre); d'autre part, la relation nouée entre le donateur d'un partenaire sexuel et la progéniture ultérieure de celui-ci (à l'instar des donneurs de femmes représentés par l'oncle maternel auprès de son neveu utérin). Pour Lévi-Strauss, les «conduites stylisées» entre parents (la gêne, l'intimité, la plaisanterie, l'hostilité, l'autorité, la familiarité...) indexent un à un ces liens et leurs variantes, et peuvent être regroupées en deux ensembles symétriquement opposés et dotés d'une valeur positive ou négative dont l'expression obéit à l'analogie proportionnelle suivante:

A) La relation entre le donateur d'un partenaire sexuel et la progéniture ultérieure de celui-ci est à

B) la relation de consanguinité intragénérationnelle entre germains ce que

C) la relation de consanguinité intergénérationnelle (entre parents et enfants, entre ascendants et descendants) est à

D) la relation d'affinité entre conjoints et/ou entre beaux-parents.

Dans sa version la plus simple, illustrée en Mélanésie chez les Trobriandais, cette «loi de l'atome de parenté» se vérifie à travers l'homologie du rapport entre l'oncle maternel et le neveu utérin, respectueux et antagoniste (-), et celui des frères et sœurs, chargé de tabous (-), vis-à-vis des rapports entre père et fils, libres et familiers $(+)$, et mari et femme, de l'ordre de l'intimité et de l'affection (+).

Système des attitudes aux îles Trobriand.

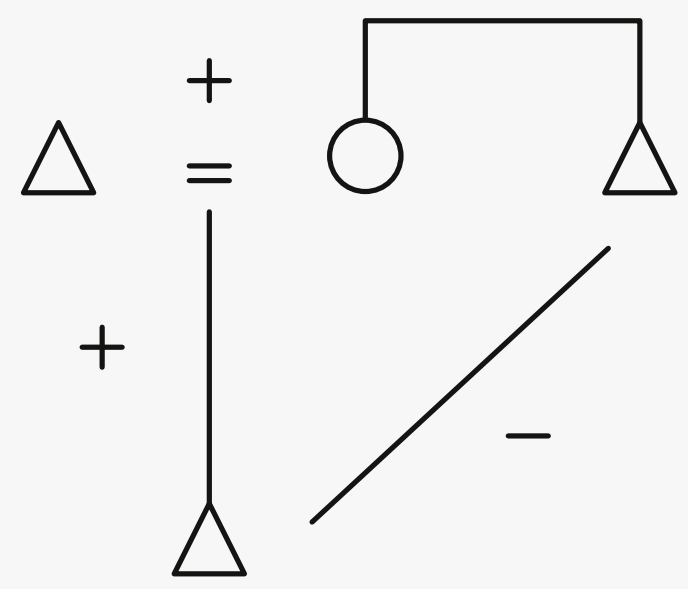


Cependant, comme le rappelle à juste titre Godelier (p. 192), LéviStrauss, face aux critiques de Luc de Heusch, a défendu aussi une variante plus complexe de cette structure chez les Lele du Kasaï, ou bien parmi les Mundugumor de Nouvelle-Guinée. Ce dernier cas est particulièrement significatif par la multiplication des catégories d'individus incluses dans cet atome de parenté, et donc la difficulté apparente à cerner les oppositions entre les attitudes regroupées par paires. En raison de l'existence d'une filiation matrilinéaire pour les hommes (le garçon appartient à la même lignée que sa mère, que le père de sa mère, etc.) et d'une filiation patrilinéaire pour les femmes (une fille appartient à la même lignée que son père, que la mère de son père, etc.), la relation de consanguinité intergénérationnelle $(\mathrm{C})$ concerne au premier chef l'intimité entre le père et la fille d'une part, et la mère et le fils par ailleurs (les uns et les autres dormant respectivement dans le même panier moustiquaire), tout en s'étendant aux membres éloignés de la même "corde» (la mère du père porte ainsi le même nom que la fille de celui-ci, tandis que le père de la mère celui du fils de celle-ci). Par opposition à cette valence positive, les relations d'affinité (D) entre époux, mais aussi entre beaux-frères qui se sont échangé leurs sœurs respectives à marier, sont marquées par une grande tension et une franche rivalité (-). De façon homologue, les relations entre frères et sœurs (B) sont décrits dans l'ethnographie de Margaret Mead sous un jour particulièrement hostile (-). Aussi, les donateurs respectifs d'un conjoint chez les Mundugumor, qui sont du point de vue symétrique des deux sexes, la sœur du père et le frère de la mère, entretiennent des relations de plaisanterie et de proximité très valorisées avec leurs neveux et nièces (+), allant de l'adoption au perçage des oreilles, et jusqu'à la scarification rituelle pour le neveu utérin.

Schéma de l'«atome lourd » de parenté chez les Mundugumor.

(+)

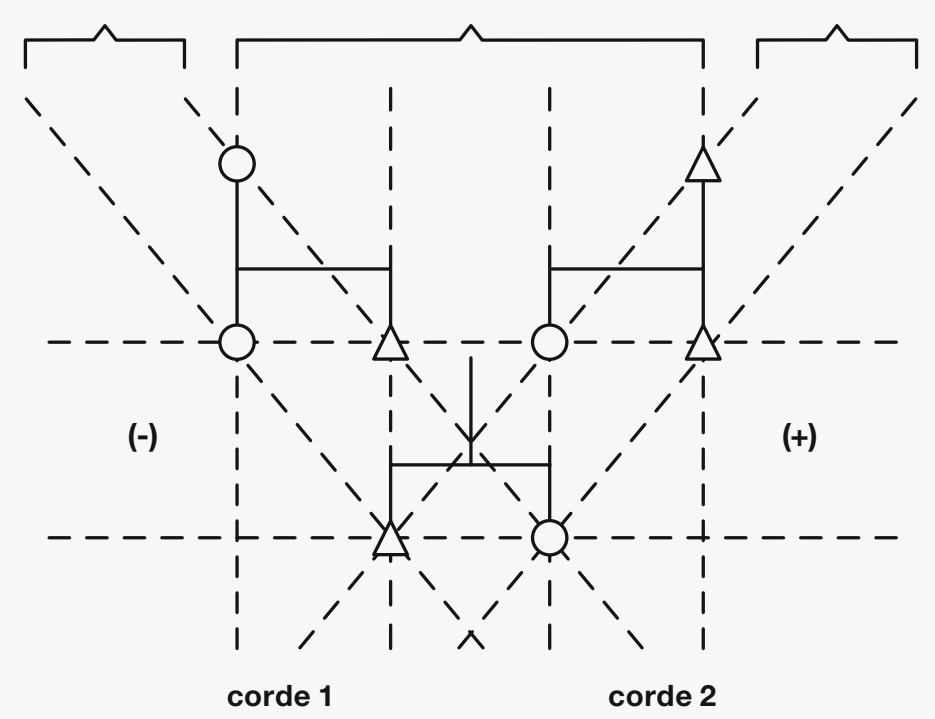

(+)
$(-)$

(+) 
2. Voir la liste établie par Maurice Godelier des cent trente oppositions binaires utilisées par Claude LéviStrauss (p. 527).

3. Ce schème mythique représente un combat entre la terre et le ciel à travers l'exil temporaire d'un héros au sommet d'un arbrerocher, d'où ce dernier descend secouru par des non-humains avec la maîtrise du feu.
On a bien là un «système» (celui des attitudes familiales) où l'organisation des comportements des parents les uns par rapport aux autres repose sur une «structure». Celle-ci (l'atome de parenté) comprend autant de transformations qu'il est possible de combiner les différentes variantes des relations de consanguinité, d'affinité et de donneur de conjoint. Et l'ensemble de ces permutations est rendu possible par une «structure cognitive " sousjacente, qui prend la forme ici d'une analogie proportionnelle (A:B::C:D) comparant deux à deux ces trois liens de parenté du point de vue de la transformation temporelle d'une relation d'affinité en relation de consanguinité.

\section{Le système mythique amérindien}

La difficulté est que cette architecture peut atteindre un degré d'organisation supérieur, à l'image de l'édifice complexe que Lévi-Strauss construit dans la tétralogie des Mythologiques pour soutenir l'existence d'un «système mythique» amérindien d'un bout à l'autre du continent américain. Est mobilisé pour cela un corpus de huit cent treize mythes soigneusement recensés par Godelier, qui relatent le temps où les humains, les plantes, les animaux, les corps célestes et les démiurges étaient indistincts, pouvaient communiquer entre eux et se métamorphoser les uns en les autres. Le premier niveau de structuration de ces récits d'origine est leur «armature». Celle-ci se compose tout d'abord de «codes», c'est-à-dire de séries d'oppositions binaires rattachées à différents registres sensoriels, culinaires, géologiques ou bien encore sociologiques (mâle/femelle, visible/ invisible, doux/amer, aigu/grave, odorant/puant, saison sèche/humide, terre/ montagne, endogame/exogame ${ }^{2}$...). On retrouve ainsi des homologies entre ces oppositions dans les mythes sur l'origine du miel et du sirop d'érable, respectivement en Amérique du Sud et du Nord. Ces séries d'oppositions se déclinent en outre sur plusieurs «axes» au sein de leur registre (un axe astronomique peut être spatial et traiter de la place des astres dans le ciel, ou bien temporel et traiter de l'apparition des astres selon les saisons).

Ce qui structure ensuite l'armature de ces mythes, ce sont les «mythèmes» et les «schèmes mythiques» qu'ils partagent (p. 389-398). Les premiers, à l'instar de la pirogue, du jaguar maître du feu, ou de la pourriture du monde, indexent dans un être ou une chose un «paquet de relations" plus abstraites pour constituer un paradigme (exemple de la pourriture comme synecdoque de l'éloignement et de la disjonction de la Terre et du Soleil); les seconds sont des scripts d'actions et d'états successifs enchaînant les mythèmes en les positionnant mutuellement sous la forme d'un syntagme narratif (à l'image du fameux voyage en pirogue du Soleil et de la Lune). Godelier expose comment, à partir de tels schèmes, LéviStrauss a parcouru «en rosace » les transformations des mythes des Bororo du Brésil chez les Amérindiens gé, tupi, warao, tacana; puis suivi celles-ci par l'intermédiaire d'un mythe tikuna jusque chez les Indiens des Plaines, de la région des Grands Lacs et du Haut Oregon, où il a retrouvé parmi les différentes populations de la côte Nord-Ouest l'ensemble des schèmes mythiques de l'Amérique du Sud relatifs à la conquête du feu, tel celui du «dénicheur d'oiseau ${ }^{3}$ ».

Les propriétés de ces armatures ouvrent sur un second niveau de structuration du système, où se jouent les transformations des mythes: 
c'est celui de la «pensée sauvage» ou des procédures de traitement de l'information du cerveau qui n'ont pas encore été reformatées par les technologies intellectuelles telles que l'écriture, l'informatique ou l'expérimentation scientifique. Il est impossible ici de présenter ces structures cognitives une à une et la manière dont Lévi-Strauss les associe pour rendre compte de la «convertibilité » et de la «médiation» des codes et des axes, ainsi que de la «combinatoire » et des «permutations », par «inversion » ou «symétrisation », de ces mythèmes et schèmes mythiques. Godelier s'y emploie d'ailleurs fort bien, même s'il n'enrichit pas sa lecture de précisions quant aux fondements cognitifs de telles opérations. Est néanmoins bien mise en valeur la façon dont la principale d'entre elles, l'analogie, fonctionne de concert avec la catégorisation des êtres et des choses, la schématisation de l'expérience, l'empathie manifestée vis-à-vis des états d'esprit d'autrui ou l'imagination de mondes fictionnels transcendant les réalités empiriques perçues.

Ainsi, pour Lévi-Strauss, si les procédures classificatoires des taxinomies végétales, minérales et animales opèrent par «opposition et corrélation», «homologie et contraste», elles tendent intuitivement cependant à «saisir l'univers sous une forme de totalité organisée " grâce au raisonnement analogique, qui infère des équivalences imaginaires entre le stade le plus abstrait et général des catégories et des éléments, et celui le plus concret et particulier des espèces et des noms. Cela est manifeste, par exemple, dans la convertibilité des catégories du haut et du bas en une opposition du ciel et de la terre, transposée à son tour dans le rapport de l'aigle à l'ours, pouvant être indexé dans la relation de deux individus singuliers représentant ces deux espèces (p. 328). De même, dans la lignée de Jean-Jacques Rousseau, Lévi-Strauss postule à l'origine du totémisme une capacité d'identification des humains aux êtres vivants, là encore issue d'une comparaison par analogie de leurs fonctions de croissance, de respiration, de nutrition et de reproduction. Le totémisme est ainsi réduit à la combinaison de deux formes d'équivalence: l'assimilation d'une personne à une plante ou un animal d'une part; I'homologie, d'autre part, des différences entre ces espèces animales et végétales du point de vue des contrastes significatifs reconnus entre les divers groupes de parenté (la relation entre l'espèce $A$ et l'espèce $B$ étant analogue à la relation entre le clan $C$ et le clan $D$, dans le sens où $A$ diffère de $B$ sur le plan de ses propriétés et de sa niche écologique tout comme $C$ diffère de $D$ sur le plan de son statut et de sa fonction dans la division rituelle du travail nécessaire à la reproduction des espèces vivantes). Quel que soit donc le domaine envisagé, l'analogie s'impose comme la structure cognitive centrale de la pensée sauvage, et Godelier ne s'y trompe pas lorsqu'il souligne la manière dont chaque volume des Mythologiques peut être parcouru comme l'exploration savante d'une facette spécifique de ce type de raisonnement inductif (p. 402-404).

Dans Le Cru et le Cuit, dédié aux «mythes paléolithiques », où sont narrées les origines du feu, de la cuisine et de la viande, Lévi-Strauss s'intéresse à la «logique des qualités sensibles» par laquelle s'établissent des correspondances entre les perceptions des cinq sens sous la forme de nomenclatures (chaud/froid, sec/humide, frais/pourri, clair/obscur, bouilli/ rôti, bruyant/silencieux...). Cette logique coïncide étonnamment avec la première des trois procédures de l'analogie reconnues dans les travaux en 
4. Voir Keith Holyoak "Analogy", in Keith Holyoak et Robert Morrison (éd.), The Cambridge Handbook of Thinking and Reasoning. Cambridge, Cambridge University Press, 2005 : 117-142.

5. Voir Mythologiques IV. Paris, Plon, 1971: 485 et 498; cité in Godelier 2013: 406. sciences cognitives aujourd'hui ${ }^{4}$, la «similarité sémantique», qui consiste à rechercher des attributs empiriques semblables pour caractériser l'homologie, par exemple, de deux termes.

Dans Du miel aux cendres, consacré aux «mythes néolithiques» traitant de l'origine de l'agriculture, c'est la «logique des formes» qui est à l'honneur dans la mise en correspondance des propriétés géométriques de transformation de l'espace (vide/plein, inclus/exclu, contenant/contenu, interne/externe). Est détaillée là aussi la contrainte de similarité sémantique, mais cette fois-ci appliquée aux relations et non aux termes (au sens où, par exemple, la calebasse comme tambour sacré est similaire à la calebasse comme récipient culinaire du point de vue de la transformation d'un arbre creux).

Quant à L'Origine des manières de table et à L'Homme nu, centrés sur le passage des mythes du sud au nord de l'Amérique, c'est précisément d'une «logique des propositions" qu'il est question: ce n'est plus la perception de similarités entre analogues qui prime, mais l'«isomorphisme structurel " des rapports existant entre leurs composantes relationnelles. Autrement dit, ce sont les relations d'opposition, d'implication, de succession, d'alternance, de fonction ou de causalité (high-order relations) entre les relations et les termes comparés (first-order relations) qui sont appréhendées. Cette appréhension se fait en vertu de la systématicité de la deuxième procédure, dite du mapping-finding, qui aligne la matrice des correspondances pour chaque niveau de structuration de l'analogue (sur le plan des attributs caractérisant les termes et les relations, sur celui du rapprochement des relations et des termes et sur celui des rapports inférés consécutifs à ce rapprochement).

La troisième procédure de l'analogie, la «centralité pragmatique», est aussi reconnaissable lorsque Lévi-Strauss assigne comme fonction à la pensée mythique de surmonter des contradictions logiques appréhendées dans la réalité empirique en les résolvant dans l'imaginaire grâce aux «déductions transcendantales». Les psychologues ont en effet bien mis en évidence la façon dont la connaissance de l'objectif associé à l'analogie influence la récupération de données en mémoire ou l'invention de représentations ajustées aux correspondances recherchées. Lévi-Strauss développe justement l'idée que la pensée sauvage infère à partir des données empiriques contrastées des faits imaginaires qui, non seulement «ne s'appuient pas sur l'expérience», mais bien souvent la «contredisent » en mettant en correspondance des phénomènes a priori très dissemblables dans le seul but de résoudre logiquement des antinomies contradictoires et irréductibles, posant problème à l'esprit humain (p. 455): «les gnomes surnaturels» en viennent de la sorte à «expliciter les traits pertinents reconnus à l'écureuil» en "greffant sur le résultat de la déduction empirique, c'est-à-dire le binarisme, une déduction transcendantale qui s'emploie à engendrer toute une imagerie qu'elle incorpore au réel, produisant un type d'évidence qu'on peut dire à bon droit apodictique ${ }^{5}$ ».

Ce passage, par l'analogie proportionnelle, de la déduction empirique à la déduction transcendantale pour l'imagination de mondes tout à la fois 


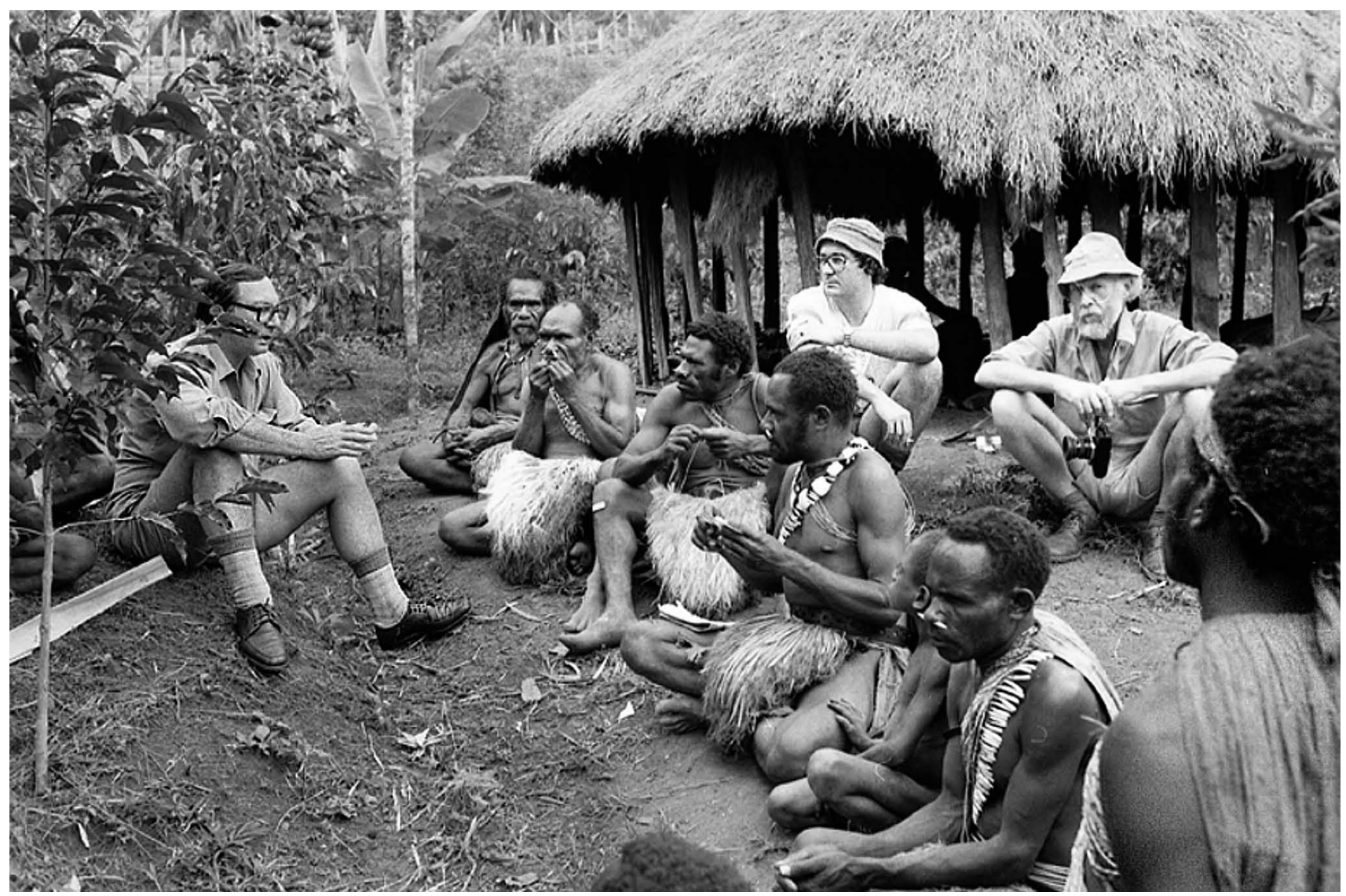

page 222 et ci-dessus

fig. 2

Quelques jours avant le début des cérémonies muka (premier stade des rites masculins) Maurice Godelier discute avec les experts rituels baruya des conditions futures de visionnage et d'utilisation des images tournées par Ian Dunlop. De gauche à droite: Maurice Godelier, Dawatnie, Tsunuandaye, Yaruemeye, Djiravenniac, Gandjakwe; en arrière plan: Jean-Luc Lory et lan Dunlop. Wonenara, juin 1979. () Pierre Lemonnier. 
6. Voir Laurent Berger, «Ritual, history and cognition: From analogy to hegemony in Highland Malagasy polities". Anthropological Theory XII (4), 2010: 351-385.

7. Voir Mythologiques IV op. cit. : 562. "Nous avons montré à maintes reprises, pour comprendre les écarts différentiels qui se manifestent dans des versions du même mythe appartenant à des sociétés voisines ou éloignées, qu'il convient de rendre à l'infrastructure ses droits. Chaque version du mythe trahit donc l'influence d'un double déterminisme: l'un la relie à une succession de versions antérieures ou à un ensemble de versions étrangères, l'autre agit de façon en quelque sorte transversale, par des contraintes d'origine infrastructurelle qui imposent la modification de tel ou tel élément, d'où résulte que le système se réorganise pour accommoder ces différences à des nécessités d'ordre externe. »

8. Voir Claude Lévi-Strauss, L'Homme nu. Paris, Plon, 1971: 314. fictionnels et réels nécessite le plus souvent d'opérer une double transformation des relations et des termes empiriques initialement comparés (le remplacement d'un terme par son contraire et la conversion d'une relation en un terme): c'est là tout l'intérêt de revisiter, comme le fait attentivement Godelier (p. 411-436), la formule canonique qui modélise ces procédures inférentielles et rend ainsi compte des rapports entre les différentes variantes d'un mythe et/ou d'un rite sur la base des transformations conditionnant l'émergence de nouveaux mythèmes, schèmes mythiques et scripts rituels ${ }^{6}$.

Cependant, la complexité de l'architecture du système mythique va bien au-delà puisqu'elle admet un troisième niveau de structuration en lien avec cette armature adossée aux structures cognitives de la pensée sauvage. Lévi-Strauss attribue en effet une place déterminante aux «infrastructures" des sociétés amérindiennes pour expliquer les différentes versions et transformations de mythes similaires en Amérique ${ }^{7}$. Seulement, et c'est là un point de désaccord central avec Godelier, ces infrastructures sont réduites aux technologies, aux connaissances et aux savoir-faire d'une part, aux ressources écologiques et aux caractéristiques astronomiques et climatiques de l'environnement par ailleurs. Autrement dit, d'un point de vue marxiste, aux seules forces productives..

Lévi-Strauss s'évertue ainsi à démontrer dans L'Homme nu que les différentes variantes du mythe de la dame plongeon à l'ouest des montagnes Rocheuses forment autant d'inversions symétriques du mythe du dénicheur d'oiseau qu'il existe de perspectives spatiales sur le territoire parcouru et mis en récit, corrélées à la répartition contiguë et l'implantation géographique sur celui-ci des tribus concernées. De la même manière, les transformations des codes et des mythèmes du sud au nord de l'Amérique sont toujours rapportées au rôle fondamental de certaines espèces, aux données climatiques et aux activités technico-économiques dans le mode de vie amérindien: par exemple, lorsque la pêche remplace la chasse, ce n'est plus la viande, mais le poisson qui prime dans l'art culinaire relaté; ailleurs, les mythes du Brésil central fondant l'origine de la cuisine sont repris dans le bassin du fleuve Columbia pour exposer l'émergence des échanges commerciaux; l'opposition du cru et du cuit cède alors la place à celle du nu et du vêtu, ou est transposée dans le passage d'une alimentation monotone à un menu quotidien diversifié... La Colombie-Britannique fournit ainsi pour Lévi-Strauss l'exemple d'un «tableau d'ensemble où s'inscrivent les valeurs et les fonctions symétriques, chaque fois liées à l'infrastructure, que le mythe du dénicheur d'oiseau reçoit chez les Klamath et les Modoc d'une part, les Salish et les Sahaptin d'autre part ${ }^{8}$ ". Les premiers, vivant loin des grandes foires commerciales et ne les fréquentant que pour vendre leurs captifs de guerre, ont ainsi ajusté son armature à l'étiologie de la chasse et des jeux de compétition intertribaux, tandis que les seconds, au cœur de l'organisation des marchés intertribaux et de la commercialisation du saumon, ont plutôt développé son armature en fonction de l'origine de la pêche et des échanges marchands.

À ce rôle prépondérant de l'infrastructure dans les configurations mythiques amérindiennes, Godelier oppose une double critique: classique 
pour ce qui concerne l'Amérique du Sud, où la réduction des infrastructures aux seules forces productives et la dénégation corrélative des rapports de production conduisent à tort au caractère plus «rudimentaire de l'organisation économique» de ces sociétés tropicales dites primitives, et donc à l'établissement de correspondances trop faciles et trop rapides entre l'armature des mythes et leur milieu technique et écologique; plus surprenante a priori pour le cas de l'Amérique du Nord, où le primat des infrastructures sur les superstructures est fortement contesté et assimilé à un matérialisme vulgaire, au nom de la marge d'autonomie irréductible de chacun des rapports sociaux constitutifs de la «société» (p. 321).

\section{D'un système à l'autre par le changement structurel}

L'enjeu d'une telle critique porte sur la façon de délimiter l'unité et de penser l'évolution historique des "systèmes" au vu de l'assemblage et des transformations des structures qui les composent. Cette prise de position de Godelier est elle-même influencée par son cheminement d'une conception marxiste du système (une formation sociale édifiée au croisement de plusieurs modes de production) à une élaboration plus contemporaine de la notion de "société", bâtie sur l'articulation de trois «rapports sociaux» types.

\section{Le progrès technique comme élément de discontinuité radicale?}

Pour sa première critique, Godelier renvoie à l'exclusion problématique, pour l'objectivation du système mythique amérindien, des cultures pueblo, navajo et zuñi, mais surtout de la mythologie des civilisations précolombiennes (inca, maya, aztèque) dont certains mythèmes, tel le jaguar, sont pourtant communs. La focalisation excessive de Lévi-Strauss sur les forces productives, au premier rang desquelles les technologies intellectuelles, induit une dichotomie radicale avec les sociétés stratifiées qui disposent de l'écriture et/ou d'un groupe de prêtres ayant systématisé sous forme théologique (panthéon) et liturgique (lieux de culte) leur mythologie. Or, si l'on prête attention en Amérique du Sud aux rapports économiques et politicoreligieux, ce grand partage radical laisse plutôt place à un continuum de formations politiques, certes hétérogènes par leur peuplement, leur taille démographique, leurs capacités militaires et leur stratification, mais symbiotiquement enchâssées dans leurs niches écologiques respectives, au gré des échanges, des conflits et des déplacements des groupes qui les animent. Ce fait repose ouvertement la question d'une continuité transformationnelle ou d'une rupture dichotomique du système mythique amérindien selon la nature étatique ou acéphale de ses sociétés constitutives. À l'appui de cette objection soulevée par Godelier peut être mobilisée la démonstration récente apportée par Fernando Santos Granero de l'existence précoloniale de régimes de servitude parmi certains groupes amérindiens des familles linguistiques tukano, tupi-guarani (les Chiriguana de Bolivie) et pano (les Conibo d'Amazonie péruvienne) présentant une similarité avec les institutions esclavagistes de la côte Nord-Ouest du Pacifique, des Grandes Plaines et du bassin du fleuve Columbia ${ }^{9}$.

Ces relations extrêmes de dépendance impliquent des raids saisonniers entre plusieurs agglomérations et populations ennemies, alliées ou tributaires, et se manifestent dans le traitement des captifs de guerre et
9. Voir Fernando Santos Granero, Vital Enemies: Slavery, Predation, and the Amerindian Political Economy of Life. Austin, University of Texas Press, 2009. 
CLAUDE LEVI-STRAUSS

MYTHOLOGIQUES

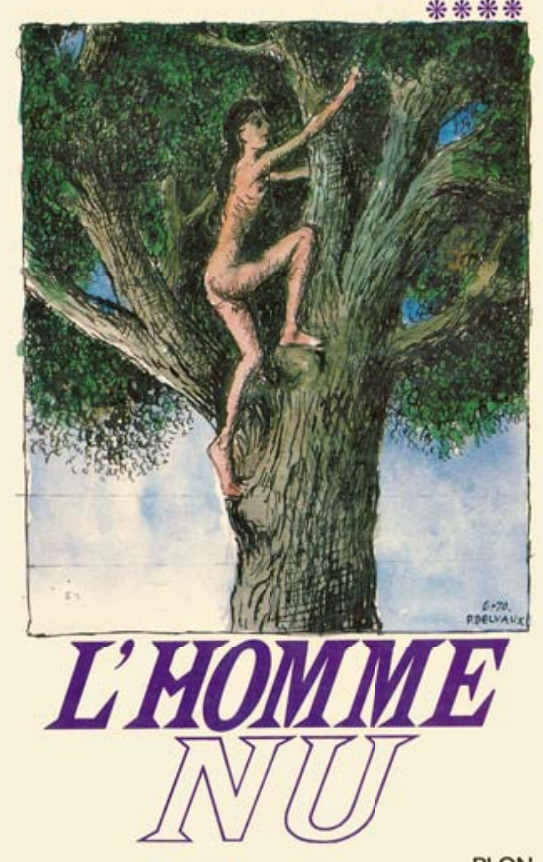

PLON

\section{GODELIER}

Métamorphoses de la parenté

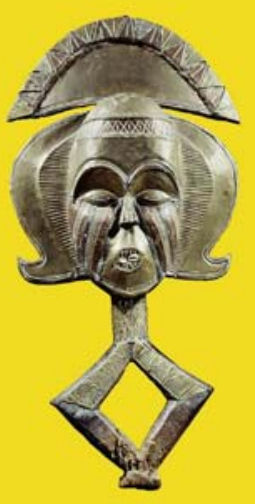

Champs essais

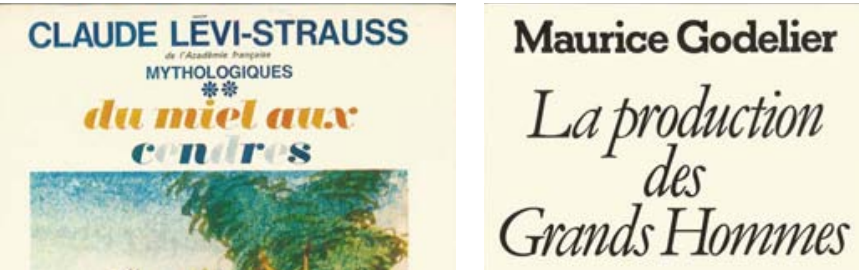

\section{LÉVI-STRAUSS} MAURICE GODELIER

SEUIL
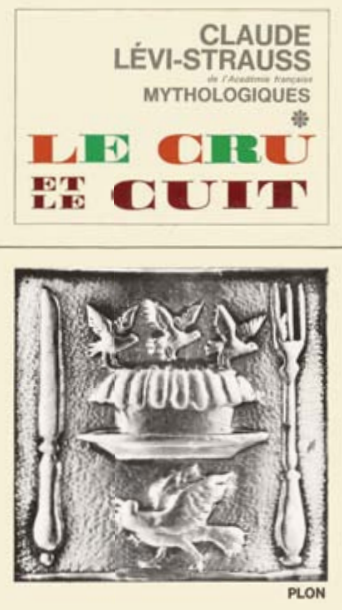
des groupes de serviteurs assignés à résidence (à l'instar des horticulteurs chané envahis par les Chiriguana ou des chasseurs-cueilleurs maku rattachés aux Tukano). Contrairement au refus d'assimiler ces captifs à de véritables esclaves, au nom de leur mise à mort rituelle ou de leur incorporation par le mariage et l'adoption aux familles de leurs ravisseurs, certaines données montrent que leur statut reste marqué du sceau de l'infériorité (par des mutilations corporelles, des tatouages faciaux, des vêtements et des coiffes, des rituels d'asservissement les dépersonnalisant en tant que dépendants génériques, des interdits et prescriptions matrimoniales stricts), et se transmet sur deux ou trois générations à travers une spécialisation permanente, saisonnière ou intermittente comme domestique, concubine, guerrier, porteur, chasseur ou horticulteur au service de son maître.

En outre, sices régimes de servitude ne sont pas une forme d'esclavage productif, au sens où ils ne libèrent pas les groupes ravisseurs de toute production directe, leur contribution à l'économie domestique, rituelle, guerrière et politique s'avère essentielle, comme l'atteste d'ailleurs le poids démographique de ces dépendants (entre un tiers et la moitié de la population chez les Tukano et les Chiriguana). Ces derniers accomplissent en effet une bonne partie des tâches ménagères et artisanales des maisonnées, souvent les plus difficiles et méprisées (les corvées d'eau, de bois et de manioc, par exemple); les hommes aident aux travaux horticoles, à la constitution de stocks alimentaires et de boissons fermentées, tandis que les femmes satisfont les envies sexuelles de leurs maîtres et des fils et hôtes de ces derniers, nourrissant au sein et veillant leurs enfants; les expéditions guerrières sont lancées avec leur concours, par leur assignation au portage de longue distance, à la conduite des canoës ou bien encore aux premières vagues d'assaut lors des combats.

Enfin, s'il n'existe pas, en dehors de la demande coloniale, de marchés aux esclaves (à l'image de leur vente contre des armes et des produits manufacturés lors du boom du caoutchouc en Amazonie en 1880), et donc une marchandisation des captifs, ceux-ci n'en demeurent pas moins la propriété aliénable et mobilière de leur maître, qui peut selon son bon vouloir les céder pour acquérir des objets cérémoniels, les faire rituellement exécuter ou les offrir en guise de présent, entre chefs de malocas tukano par exemple. Leur valeur réside en effet non dans la richesse produite, mais dans le temps qu'ils libèrent et les services qu'ils rendent, au rang desquels prévaut l'indexation relative du prestige et de la puissance des chefs et guerriers dans cette économie politique de conquête de la vitalité d'autrui. Ce qui prime est, au-delà de la réduction des ennemis à des proies chassées, transformées en victimes immolées ou en êtres apprivoisés, éventuellement assimilées à des parents, leur intégration comme captifs de guerre et serviteurs, et leur recyclage comme "potentialités de vie», transférées et accumulées aux dépens d'autrui. Autrement dit, ces régimes de servitude sont les chaînons manquants pour penser l'émergence précolombienne des formations politiques stratifiées et centralisées en Amérique du Sud tropicale, par la libération partielle des tâches productives et la spécialisation guerrière conséquente d'une élite entourée d'une suite de dépendants que ces régimes sont susceptibles d'entraîner.

\section{ci-contre}

fig. 3

Couvertures des quatre tomes des Mythologiques de Claude Lévi-Strauss, Plon, 1964-1971 et des éditions suivantes des ouvrages de Maurice Godelier: La production des Grands Hommes, Fayard, 1996 ; L'énigme du don, Flammarion, 2008; Métamorphoses de la parenté, Flammarion, 2010 et Lévi-Strauss, Seuil, 2013. D.R 
10. Voir Maurice Godelier, La Production des grands hommes. Pouvoir et domination masculine chez les Baruya de Nouvelle-Guinée. Paris, Fayard, 1982

\section{Les infrastructures à l'origine du changement historique?}

La seconde critique émise par Godelier vise l'hypothèse de causalité en dernière instance des infrastructures que reprend à son compte LéviStrauss («dans le sens de ce qui provoque et amène à la pensée des situations contradictoires") lorsqu'il se défend, par exemple, dans La Pensée sauvage, de ne contribuer qu'à une "théorie des superstructures". Cette critique se développe en trois points: le premier est la défense de l'autonomie relative des trois rapports sociaux, posés comme des structures complémentaires formant système, au fondement de toute société humaine; le deuxième est l'attribution aux rapports politico-religieux d'un rôle majeur dans l'articulation et la totalisation synchronique de ces rapports autonomes et interdépendants en une société singulière; le troisième est la reformulation de la distinction infrastructure/superstructure à partir du rôle dominant joué par les rapports de production dans l'évolution diachronique de ces formations politiques.

La notion de «rapport social» est centrale chez Godelier en ce qu'elle étend celle de structure à des domaines négligés par Lévi-Strauss, tout en plaçant les relations de pouvoir en son cœur. Un rapport social désigne en effet un ensemble de relations entre des positions occupées par des individus et des groupes, organisé à partir de l'allocation et de l'appropriation de ce qui fonde pour chaque rapport un enjeu de lutte constitutif de son existence même; à savoir, pour les "rapports de parenté", les moyens de procréation (usages des sexes, PMA, GPA...), les conjoints potentiels, la progéniture mise au monde, les foyers domestiques...; pour les «rapports économiques ", les moyens de production et de transaction (force de travail, techniques, connaissances, matières premières, monnaies), les produits du travail, la répartition des tâches dans les procès de travail...; et pour les «rapports politico-religieux», les moyens de communication et de destruction (médias, rituels, armes), ainsi que le mode de gouvernement et de souveraineté à l'égard d'autrui et d'un territoire riche en ressources utilisables. Dans cette perspective, il n'existe pas de «fondations» nécessaires à cet "édifice» qu'est un "système social»: chacune de ces trois «structures" est indispensable par leur articulation conjointe à l'existence de ce dernier.

Godelier conteste ainsi l'idée prégnante depuis Lewis Henry Morgan - et faite sienne par Lévi-Strauss - que les "structures » de parenté forment la base organisationnelle des sociétés dites primitives et tribales. $\mathrm{Si}$, par exemple, chez les Baruya, les relations d'alliance et de descendance encadrent les formes de coopération, l'appropriation des moyens de production et le partage des produits, eux demeurent subordonnés à la domination des hommes sur les femmes instituée par les initiations masculines ${ }^{10}$. Godelier dénonce ainsi, à la suite d'Edmund Leach, la vision lévi-straussienne d'un système kachin bouclé sur lui-même par les cycles longs de l'échange généralisé, où tous les groupes de descendance s'ordonneraient les uns aux autres à la suite de mariages prescriptifs avec la fille de l'oncle maternel grâce à la supériorité garantie des donneurs sur les preneurs de femme. De même, il nuance l'analyse des "systèmes à maisons", bâtie exclusivement sur cette structure de parenté, en montrant que les critères du choix du conjoint, de la résidence et de la filiation ne sont pas «subvertis" par "des intérêts politiques et économiques tendant à envahir le champ social» 
sans pour autant disposer d'un «langage distinct de celui de la parenté ${ }^{\mathbf{1 1}}$ ». Certes, la manipulation des règles de succession, les manœuvres familiales hypo/hyper-gamiques pour se marier au proche et au loin, la pratique ciblée de l'adoption et de la résidence commune diversifient autant que faire se peut les stratégies de perpétuation des maisons dans le temps en favorisant leur monopolisation et leur exploitation de ressources nouvelles d'extraversion ou bien leur accumulation intergénérationnelle de biens domaniaux, de rangs et de titres. Cela constitue évidemment des moyens importants pour construire une supériorité hiérarchique associée à la genèse et au développement de chefferies aristocratiques et d'États archaïques. Cependant, rappelle Godelier, l'importance des alliances matrimoniales est la plupart du temps supplantée par celle des alliances politiques et militaires, tout comme les conditions d'exercice du pouvoir et la reconnaissance du rang élevé de certaines maisons relèvent en priorité de l'organisation de rituels et de grandes cérémonies redistributives, ainsi que de pratiques guerrières et gouvernementales, qui trouvent d'abord à s'exprimer et se légitimer dans un langage religieux (la maison impériale au Japon est ainsi supposée descendre de la déesse du riz).

C'est pourquoi Godelier prend ses distances avec le déterminisme infrastructurel auquel souscrit Lévi-Strauss par le biais du positionnement des structures de parenté à la base de certaines sociétés ou par l'importance conférée aux forces productives, enrichies de leurs opérations cognitives. On connaît à ce titre la thèse défendue dans L'ldéel et le Matériel (1984: 194) selon laquelle la différence entre infrastructure et superstructure ne peut être une distinction d'instance ou de sphère d'activité. Seule compte en effet la «fonction» remplie par ces institutions, en l'occurrence celle d'allocation et d'appropriation des produits du travail et des moyens de production et de transaction ${ }^{\mathbf{1 2}}$. Ce que l'on connaît moins bien peut-être, c'est le cadre évolutionniste à partir duquel cette thèse reste compatible avec l'idée précédente de l'importance fondamentale du pouvoir souverain et des rituels collectifs pour la stratification et la totalisation des sociétés humaines. Pour Godelier, en effet, le contrôle monopolistique des moyens de production par une classe, une caste ou un ordre n'est possible que si ces minorités s'arrogent auparavant le contrôle des conditions imaginaires de reproduction de l'univers et de la vie grâce à leur monopolisation des moyens de destruction et de communication (notamment rituels), persuadant le plus grand nombre du bien-fondé à leur procurer des biens et des services matériels en échange d'une garantie de vitalité, fertilité, fécondité et prospérité. En d'autres termes, le déplacement des luttes de pouvoir de la répartition des produits du travail vers l'appropriation des moyens de production est corrélatif du développement des forces productives et de l'importance relative prise alors par les rapports économiques.

\section{Des systèmes de parenté distincts ou structurellement variables?}

Ce passage des modes de production à la société pour comprendre la structuration des formations politiques a des conséquences importantes sur la place dévolue aux «rapports de parenté». Ceux-ci se trouvent à la fois relativement autonomes et solidaires des autres rapports sociaux. Leur changement historique peut ainsi obéir à des logiques et des temporalités singulières, qui sont indépendantes, pour une large part, de celles
11. Voir Claude LéviStrauss, Paroles données. Paris, Plon, 1984: 191.

12. "Pour qu'une activité sociale - et avec elle les idées et institutions qui lui correspondent et l'organisent - joue un rôle dominant dans le fonctionnement et l'évolution d'une société, il ne suffit pas qu'elle assume plusieurs fonctions, il faut nécessairement qu'elle assume directement, en plus de sa finalité et de ses fonctions explicites, la fonction de rapport de production. " 


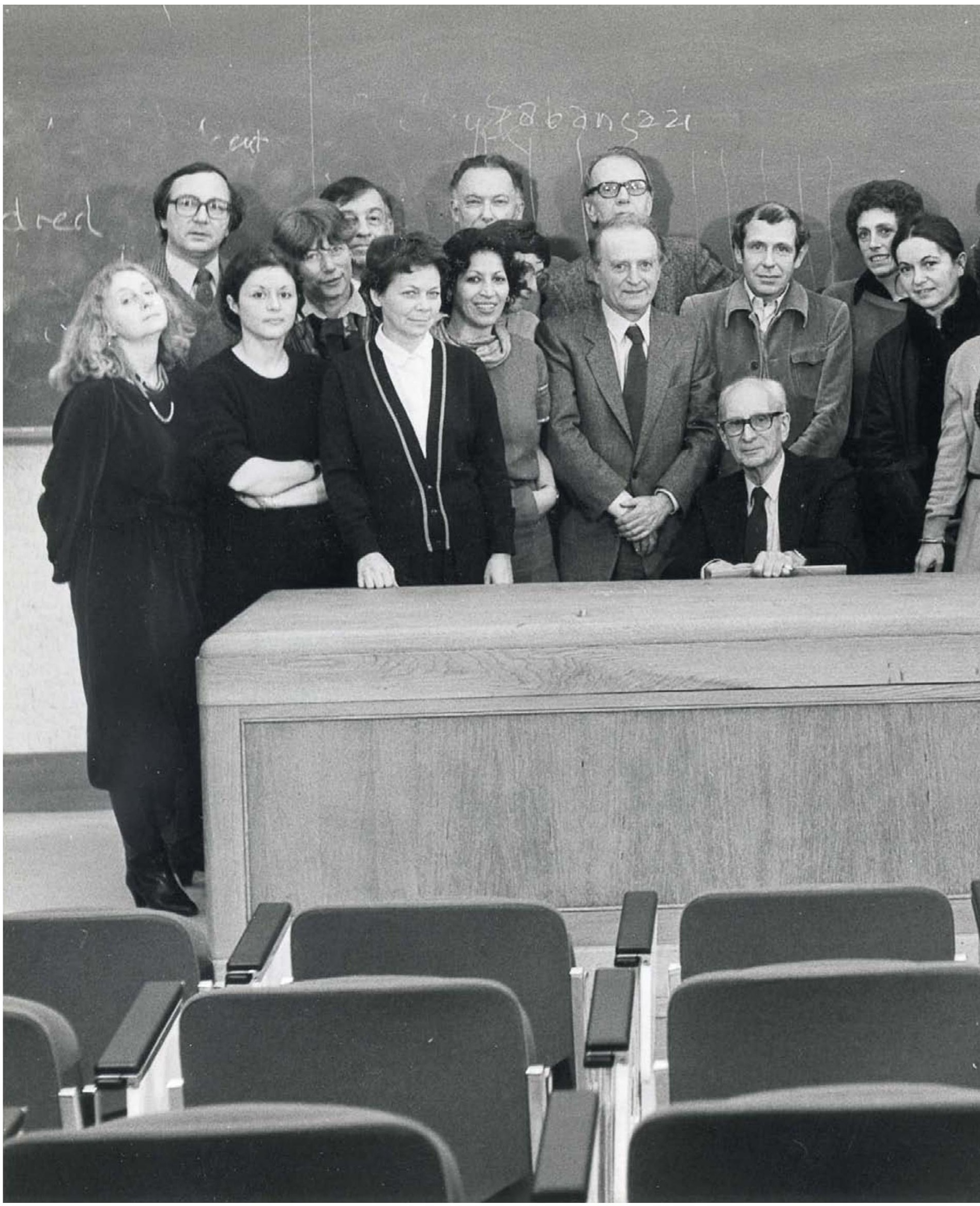




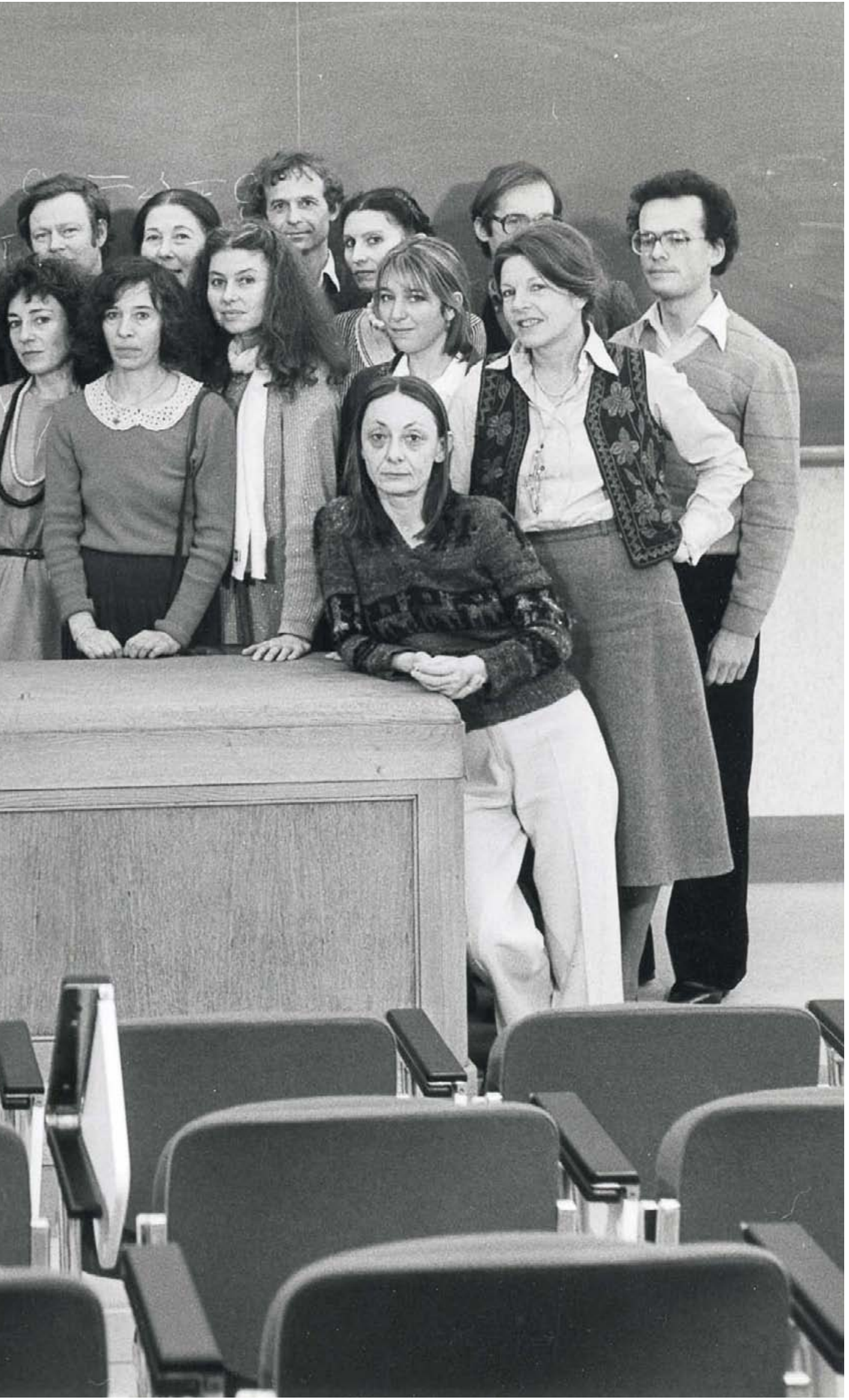

fig. 4

Dernière leçon de Claude Lévi-Strauss au Collège de France, 1983. Archives Maurice Godelier, D.R. 
13. Maurice Godelier Métamorphoses de la parenté. Paris, Fayard, 2004: 528. qui entraînent la transformation des rapports économiques et politicoreligieux (à l'instar de l'évolution des terminologies), tout comme ces derniers peuvent être amenés à les pénétrer et les subordonner à leur reproduction en imprimant les rôles et les statuts qu'ils offrent à chaque individu, sous la forme par exemple d'attributs de genre (masculin/féminin) dans les fonctions parentales et les corps sexués ${ }^{13}$. Lorsque Godelier envisage ainsi la systématisation des rapports de parenté chez Lévi-Strauss, c'est pour souligner la façon dont celui-ci s'est fourvoyé en soutenant l'existence d'un "système de parenté archaïque" commun à l'Inde et la Chine fondé sur l'échange généralisé, ou bien en assimilant les composantes crow-omaha de certains rapports de parenté à l'émergence de structures semi-complexes, alors que ceux-ci semblent se rattacher à des structures élémentaires différenciées par une variante supplémentaire de l'échange généralisé.

Pour ne prendre que le cas de l'Inde, les travaux de Louis Dumont, Robert Parkin et Gloria Raheja ont depuis documenté le fonctionnement distinct, associé respectivement aux groupes de langues dravidiennes, austronésiennes et indo-européennes, de la parenté tamul (échange restreint de type dravidien), munda (variante d'un échange généralisé) et indo-aryenne (structure complexe basée sur la dot et le don d'une vierge), interdisant de fait toute possibilité de les confondre en un seul système (p. 111).

Quant aux recherches contemporaines de Thomas Trautmann, elles mettent en évidence la façon dont les "systèmes crow-omaha", caractérisés par le principe de fusion oblique, l'absence de règle prescriptive de mariage et la multiplication corrélative des interdits matrimoniaux visant certains groupes de descendance et positions généalogiques, dérivent historiquement et logiquement de formes iroquoises, une fois disparus l'échange direct des femmes et le mariage entre cousins croisés à $\mathrm{G}^{0}$, mais conservée l'idée d'un renouvellement des mêmes alliances sur plusieurs générations (p. 166-176). Partir de la structure élémentaire des systèmes dravidiens pour établir une série de variantes au sein d'un seul groupe de transformation, dont les formes iroquoises précèdent et produisent par dérivation celles dites crow-omaha, s'impose aujourd'hui ainsi comme I'hypothèse la plus plausible. Celle-ci est d'ailleurs confirmée par le rôle que jouent les terminologies crow-omaha dans d'autres rapports de parenté qui, soit combinent une règle prescriptive de mariage et une règle prohibitive d'alliance avec deux catégories différentes de cousins croisés (c'est le cas des systèmes asymétriques prescriptifs de type kachin ou murngin, exclus à tort par Lévi-Strauss de ce groupe de transformation), soit utilisent le principe de fusion oblique pour garantir à des groupes de descendance unilinéaire la transmission exclusive en leur sein de droits, de privilèges et de responsabilités politiques et rituelles (c'est le cas de la variante iroquoise matrilinéaire décrite par David Kronenfeld chez les Fanti au Ghana).

Si les faits et les analyses mobilisés par Godelier remettent en cause l'existence même des "structures semi-complexes", ils n'en valident pas moins l'intérêt à distinguer et opposer les structures élémentaires et complexes de la parenté. Car c'est en se focalisant sur les conditions du passage des premières aux secondes que Godelier développe et confirme, à partir du cas mélanésien, l'idée de Lévi-Strauss selon laquelle l'introduction 
dans le mariage de compensations matrimoniales est l'innovation qui ouvre à la transformation de l'échange généralisé en structure complexe, où le choix du conjoint est motivé par des raisons personnelles, religieuses, politiques ou économiques (p. 116). Lévi-Strauss décrit cette transition dans les sociétés agropastorales de l'Afrique nilotique et australe, où le bétail reçu en échange d'une femme permet au frère de celle-ci de se marier en transférant à son tour à la famille de sa future épouse ces têtes de bétail, sans qu'il y ait de prescription catégorielle du conjoint. Dans la cinquantaine de sociétés mélanésiennes comparées par Godelier, ce sont les cochons et non le bétail qui peuvent être utilisés comme monnaie primitive pour le paiement de ces compensations matrimoniales. Lorsque l'échange des sœurs est alors abandonné pour s'acquitter du prix de la fiancée, Godelier soutient que cette innovation va de pair avec la production et l'accumulation de richesses, ainsi que l'organisation pionnière d'échanges cérémoniels agonistiques de type potlatch remplaçant les guerres intertribales (à l'instar du tee chez les Enga ou du moka chez les Melpa). On ne passe pas alors seulement d'un système de parenté à l'autre, mais d'un type de société à l'autre à travers différentes variantes transitionnelles, au terme desquelles les great men et les initiations masculines font place aux big men et aux cultes des déesses de la fertilité. Mais pour que cette évolution historique ait lieu, il faut à la fois une structuration précise des rapports politico-religieux et une transformation des structures cognitives sous-jacentes à l'ensemble des rapports sociaux constitutifs de ces formations politiques tribales.

Pour Godelier, il est en effet nécessaire que leur organisation exclue toute forme de monopole par certains groupes de descendance des moyens de procréation, de production, de transaction, de communication et de destruction (en d'autres termes, que la hiérarchie statutaire des groupes et des individus ne soit pas héréditaire, mais ouverte à la compétition). La seconde condition est qu'une véritable «révolution mentale " s'opère à travers la mise en pratique d'un nouveau schème conceptuel et d'un nouveau principe d'équivalence établi par analogie pour obtenir des épouses, établir des alliances diplomatiques, gérer l'homicide d'ennemis et d'alliés ou communiquer avec les esprits et les divinités: il faut, d'une part, le remplacement du don réciproque par le don agonistique (lorsqu'il est donné plus qu'il n'est reçu pour affirmer sa supériorité et instaurer une dissymétrie dans la relation) et, d'autre part, la substitution de richesses à la place de vies humaines (lorsque le renoncement à une femme ou à un guerrier mort au combat peut désormais se compenser par des biens de valeur homologues ${ }^{14}$ ).

\section{Du déterminisme structurel à l'agentivité conjoncturelle}

Pour comprendre la place faite par Godelier aux événements et à l'agentivité humaine dans ces transformations historiques, il est important de saisir la nature si particulière de ces structures cognitives, ainsi intercalées entre le changement des rapports sociaux (la praxis) et l'activité sociale qui s'y déroule (la pratique ${ }^{15}$ ).

Une capacité d'action structurée par un schématisme conceptuel

Pour Lévi-Strauss, ces structures cognitives sont des «schèmes conceptuels ", à l'interface de l'inné et de l'acquis, dont le prototype est la relation symétrique par réciprocité. Pour reprendre l'expression de Pierre
14. Voir Maurice Godelier, L'Énigme du don. Paris, Fayard, 1996: 219.

15. Voir La Pensée sauvage, op. cit.: 160. 


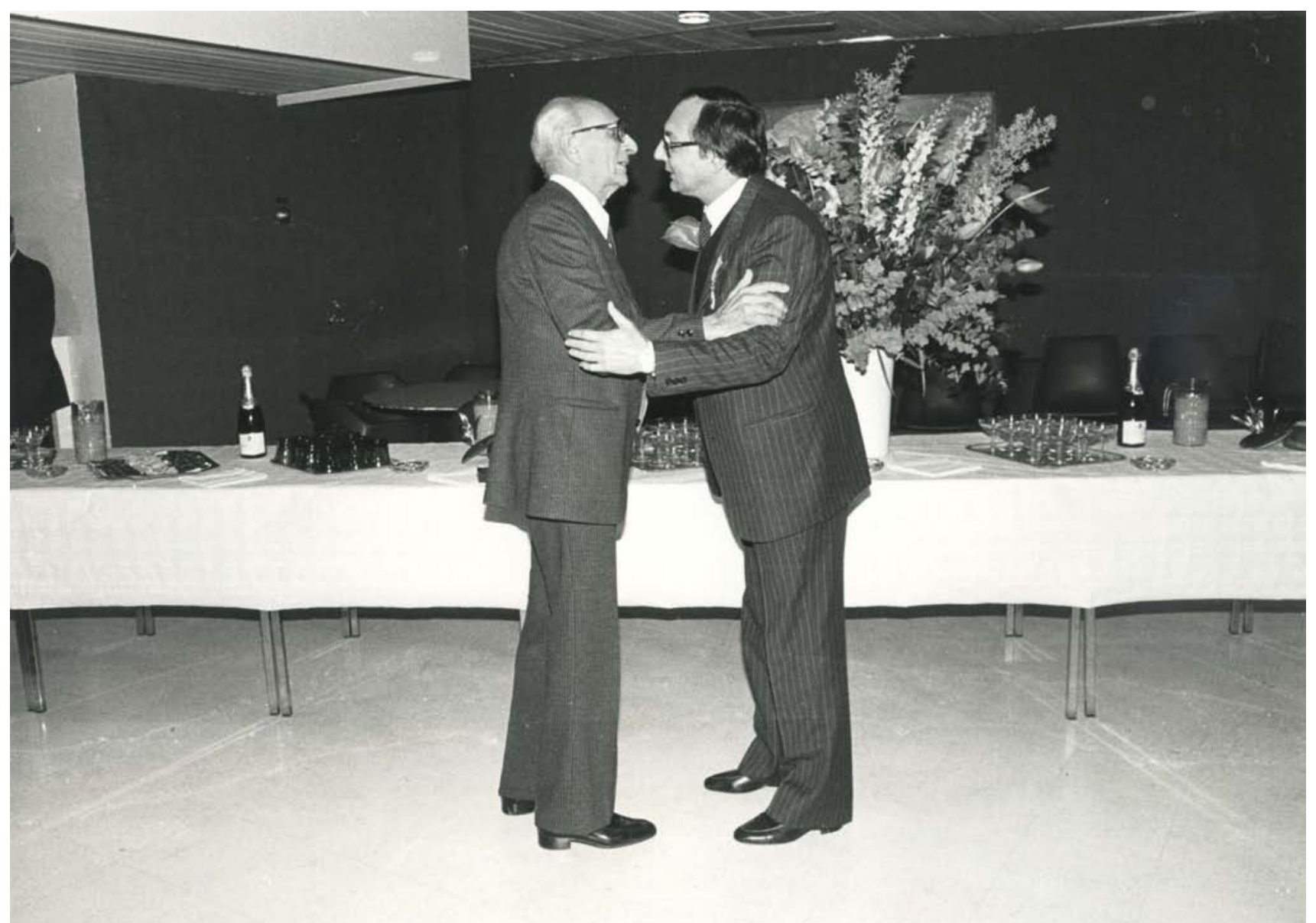

fig. 5

Maurice Godelier avec

Claude Lévi-Strauss, s.d.

Archives Maurice Godelier,

D.R. 
Bourdieu, ces «structures structurées prédisposées à fonctionner comme structures structurantes » constituent des dispositions à l'interaction tacites, durables et transposables d'un domaine de la vie sociale à l'autre, dont la forme est prédéterminée par un traitement parallèle et distribué de l'information, mais dont le contenu est appris et mémorisé au gré d'expériences vécues, partagées et répétées. Godelier prend le risque d'étendre cette notion aux trois prédispositions stratégiques qui orientent selon lui la façon de s'approprier et d'allouer ce qui fonde l'enjeu de lutte de chaque rapport social: la possibilité de garder pour conserver ou transmettre (en s'arrogeant l'exclusivité des droits de propriété et d'usage), celle de donner pour partager ou endetter (en cédant les droits d'usage pour maintenir certains droits de propriété) et celle d'échanger pour se développer ou décroître (en transférant les anciens droits de propriété et d'usage afin d'en acquérir de nouveaux, plus ou moins importants). Reprochant à Lévi-Strauss une conception du lien social restreinte à la mise en circulation des êtres humains, des biens et des idées, il met au contraire en avant les variantes que peuvent revêtir ces trois schèmes conceptuels fondamentaux (par exemple don réciproque/agonistique), dont la combinaison en proportion variable dans chaque rapport social ouvre à la conduite, en contexte historique, de «politiques de la valeur» esquissant ce qui, dans la pratique, doit demeurer ou être soustrait à la circulation comme support durable d'identification et d'appartenance (option de la patrimonialisation), ou bien à l'inverse entrer dans l'échange administré ou conditionné par l'offre et la demande (options de la redistribution et de la marchandisation).

L'allocation et l'appropriation de la monnaie dans les rapports économiques en sont une bonne illustration puisque ce ne sont pas la quantité et le type de travail nécessaires à sa fabrication qui fixent sa valeur, comme le montre la production de sel végétal chez les Baruya ${ }^{16}$. Ces barres de sel peuvent être en effet gardées comme des objets sacrés afin d'être utilisées dans les initiations comme équivalent du sperme et pourvoyeuses d'une puissance divine aux jeunes générations masculines. Mais elles peuvent aussi être mises en circulation comme objets précieux (valuables) au sein de la tribu en étant exclusivement données aux affins et aux cousins croisés pour honorer des obligations sociales, tout comme elles sont commercialisées et fonctionnent comme unités de compte au sein des échanges intertribaux régionaux afin d'acheter les armes, les denrées alimentaires, les parures et les ornements qui manquent aux Baruya sur leur territoire. Cependant, l'exemple le plus saillant, pour ce qu'il révèle des limites de la théorie lévi-straussienne de la prohibition de l'inceste, est encore une fois celui des rapports de parenté.

Godelier se démarque de Lévi-Strauss à ce sujet sur deux points cruciaux. Le premier est la prise en compte de toutes les formes de circulation possibles des personnes entre les foyers domestiques et les groupes de parenté. Non seulement l'alliance matrimoniale n'en est qu'une modalité, non seulement celle-ci se décline selon des logiques de don et d'échange spécifiques, mais de surcroît, comme l'atteste le cas de sociétés matrilinéaires, uxorilocales ou matrilocales, ce ne sont pas seulement les femmes qui peuvent faire l'objet de transactions entre hommes, mais aussi les hommes entre femmes, et les personnes entre groupes ${ }^{17}$. II existe tout
16. Voir Horizon, trajets marxistes..., op. cit.

17. Godelier critique sévèrement Lévi-Strauss quand celui-ci ancre en 1949 cette circulation à sens unique dans la tendance innée à la polygamie, et le développement du cerveau et de l'aptitude au langage. 
un éventail de pratiques maritales où ce peut être le don réciproque de sœurs réelles/classificatoires entre frères ou d'individus de sexe différent entre maisonnées qui prédomine (avec à la clé dans ce dernier cas l'égalité des donneurs et preneurs de conjoint); où, en revanche, c'est le don agonistique d'une partenaire conjugale entre groupes de descendance qui prime, lorsque le prix de la fiancée sanctionne la supériorité des donneurs sur les preneurs; où c'est plutôt une forme d'échange administré entre les deux familles qui prévaut quand la dot accompagnant la future épouse scelle, comme en Inde, la centralité du brahmane et la supériorité des preneurs par leur prise en charge rituelle de la fertilité polluante de la jeune vierge menstruée; et où, enfin, c'est l'échange marchand qui s'impose dès lors que les richesses utilisées comme compensations matrimoniales subissent une monétarisation et connaissent une fluctuation de leur valeur conséquente à leur insertion dans l'économie de marché, voire lorsque les compagnes sont acquises par le biais de la traite esclavagiste.

Le second point est complémentaire du premier: les rapports de parenté ne sont pas réductibles aux relations d'alliance parce qu'ils ne se limitent pas à la mise en circulation de conjoints potentiels et parce que la prohibition de l'inceste ne se restreint pas à l'obligation de céder sa mère, sa sœur ou sa fille à autrui pour se coordonner avec lui. À l'appui de ce contrepoint, il y a l'absence de vie maritale chez les $\mathrm{Na}$ et les Nayar et l'union sexuelle et matrimoniale des frères et sœurs dans la Perse mazdéenne et l'Égypte pharaonique et ptolémaïque. Ces deux cas illustrent la possibilité pour les foyers domestiques et les groupes de parenté de ne pas se donner ou échanger des partenaires conjugaux, mais de garder et conserver ces derniers en leur sein. L'enjeu peut en effet concerner tout autant l'appropriation de la progéniture et l'allocation des fonctions de parentalité (engendrement, élevage, éducation, protection, autorité...) quand la transmission et le partage de noms, de connaissances, de statuts et de biens mobiliers et immobiliers sont susceptibles de déterminer la façon dont les positionnements des individus et des groupes les uns par rapport aux autres se reproduisent et perdurent, génération après génération. Pour Godelier, «Lévi-Strauss minimise le fait que ce qui compte pour les familles dans l'alliance, c'est qu'elles continuent d'exister à travers leurs descendants nés de cette alliance ", que ce soit en les gardant auprès d'elles pour se perpétuer ou en les cédant et les échangeant pour se développer (p. 442). C'est pourquoi, à ses yeux, toute théorie de la prohibition de l'inceste implique de tenir compte complémentairement de l'alliance et de la descendance car cette prohibition ne dérive pas seulement de la nécessité pour les familles consanguines de pratiquer l'exogamie (marrying-out or being killed out), mais aussi de l'impératif à exercer au sein des foyers domestiques un certain nombre de responsabilités parentales ainsi qu'une forme de coopération dans la division sexuelle du travail entre les générations, toutes apparues avec la domestication du feu et l'invention des unités de procréation et d'élevage.

\section{Une réflexivité critique relevant de la métacognition}

La mise en pratique alternative de schèmes conceptuels tels que la transmission, le don ou l'échange, dans leurs multiples variantes, est selon Godelier ce qui oriente historiquement le changement social et culturel, 
c'est-à-dire ici la structuration différenciée des rapports sociaux. Pour autant, certaines composantes matérielles et idéelles de la parenté, de l'économie ou du politique et du religieux peuvent apparaître ou disparaître sans que la dominance hégémonique de l'un de ces schèmes par rapport aux autres, dans un domaine ou dans l'ensemble de la société, soit altérée. II faut par conséquent aller au-delà de la répartition lévi-straussienne des tâches entre l'histoire, l'ethnologie et la psychologie: la première ne peut se contenter des actions contingentes, dont les conditions d'occurrence seraient balisées par l'analyse structurale des groupes de transformation virtuels; la troisième ne peut se satisfaire d'une cognition élémentaire et implicite, réduite à l'analogie et aux schèmes, qui abandonnerait les plus hautes créations de l'esprit et les fonctions de la pensée à l'herméneutique ethnographique et historiographique.

Godelier rejoint ainsi la critique d'Emmanuel Terray quant au rôle limité dévolu à l'histoire par le structuralisme: rendre compte de la réalisation située et datée d'une variante appartenant à un groupe de transformation n'explique en rien le «passage du possible au réel ». C'est en dernière instance par l'entremise d'un choix optionnel que ce passage s'opère, et cette prise de décision est ce qui fonde l'art de la politique et le cours événementiel de l'histoire. Ceci entraîne du coup une réévaluation du rôle assigné à la psychologie: si la pensée humaine a bien pour fonction de se représenter ce qui est possible et ce qui demeure impossible (en interprétant et en rendant présentes à l'esprit les réalités extérieures et intérieures à l'organisme, en identifiant la nature, l'origine et le fonctionnement de ces réalités, en en déduisant des principes d'action ${ }^{\mathbf{1 8}}$ ), elle relève aussi de la faculté d'imagination et de la capacité à légitimer ou subvertir l'organisation des rapports sociaux en se représentant la manière dont l'impossible, sous certaines conditions, est en mesure d'advenir et de faire partie de l'ordre du possible (par l'appréhension et l'engendrement de relations d'équivalence et de nonéquivalence entre ces réalités, mais aussi par l'invention de rapports entre ces relations, dotés d'une valeur de vérité indépendante de leur perception). Dans les deux cas, la réflexivité critique est d'une importance centrale pour réguler ce double fonctionnement; c'est d'ailleurs dans les lobes frontaux du cerveau que l'on trouve cette «structure» de la pensée humaine qui, depuis les travaux de John Flavell, est dénommée «métacognition». Celle-ci consiste pour l'essentiel à connaître et contrôler le bon déroulement de la cognition pour mieux évaluer et ajuster ses apprentissages et ses résultats. Cela se fait, d'une part, en représentant sous différents formats l'information relative aux procédures cognitives (metacognitive knowledge) et, d'autre part, en supervisant de manière explicite, avec attention, la performance des tâches (metacognitive regulation) relatives à la planification des actions, à la résolution de problèmes, à la mémorisation, à la catégorisation, au raisonnement ou bien encore aux délibérations et prises de décision ${ }^{\mathbf{1 9}}$.

Comme le suggère Godelier (p. 176-185) en s'appuyant sur les travaux de Mervyn Meggit et Laurent Dousset, il est ironique que ce soit ce double angle mort de l'anthropologie structurale vis-à-vis de l'histoire et de la psychologie qui ait empêché Lévi-Strauss de résoudre, pour l'Australie aborigène, l'énigme du statut «aberrant» des systèmes de parenté aluridja (ne comportant aucune section ou sous-section, à la différence des systèmes
18. Voir Maurice Godelier, L'Idéel et le Matériel. Paris, Fayard, 1984: 199.

19. Voir Janet Metcalfe et Arthur Shimamura (éd.), Metacognition: Knowing about Knowing. Cambridge, MIT Press, 1994. 
kariera et aranda) et, par extension, le problème de l'antériorité historique ou non des premiers vis-à-vis des seconds. Une telle résolution supposait néanmoins des données empiriques indisponibles à l'époque, mais aussi surtout d'articuler ces rapports de parenté aux structures économiques et politico-religieuses. Sur de telles bases, trois avancées majeures dans la compréhension des sociétés aborigènes ont depuis été réalisées. Tout d'abord, cette «aberration » de la parenté australienne disparaît à considérer la nature dravidienne de ces systèmes, qui sont bâtis sur deux moitiés générationnelles endogames fonctionnant comme des groupes cérémoniels distincts en charge des rituels célébrant les êtres du temps du rêve. Ensuite, les systèmes kariera (à quatre sections) et aranda (à huit sections) ne s'avèrent pas seulement organisés autour de la distinction de classes matrimoniales (épousables/non épousables). Ils le sont aussi autour du rassemblement de catégories de parents en groupes totémiques complémentaires, performant respectivement les uns pour les autres les initiations et les rites de multiplication des espèces vivantes dans une division rituelle du travail élaborée à l'échelle territoriale des bandes de chasseurs-cueilleurs (celles-ci étant composées de membres appartenant à plusieurs sections, et donc en mesure de se déplacer d'un site sacré à l'autre le long des itinéraires des héros totémiques mythiques). Enfin, les systèmes dravidiens dits aberrants ont partout précédé en Australie l'émergence et la diffusion historique des systèmes à sections et sous-sections: la reconstitution par les linguistes, depuis le premier millénaire, des espaces de circulation des noms de section dans des centaines de langues aborigènes montre leur propagation le long des routes du commerce intertribal depuis la côte sudouest, sur le littoral, vers le désert central et le nord du continent jusqu'à leur introduction durant l'entre-deux-guerres dans le désert occidental chez les Pintupi et les Ngaatjatjarra, en situation de contact avec d'autres groupes aborigènes dans les stations ouvertes par l'administration coloniale.

La leçon tirée par Godelier de la conjonction de ces trois faits mérite ici d'être soulignée pour ce qu'elle révèle de la place centrale allouée à la métacognition dans son bilan critique du structuralisme. L'invention de la division en sections et sa diffusion progressive à l'ensemble du continent australien correspond selon lui à la mise en place réfléchie d'une nouvelle structure politico-religieuse, dérivée du dreamtime, qui subsume les rapports de parenté dravidiens pour les transformer radicalement. Cette innovation permet en effet aux membres de bandes et de groupes aborigènes toujours plus nombreux, et en interaction fréquente sur des territoires partagés ou adjacents, de se coordonner en se positionnant les uns vis-à-vis des autres, notamment pour organiser de grandes cérémonies totémiques au service de tous, grâce à la simplification de leur répartition aux différents lieux et moments des cycles rituels, calculée antérieurement en fonction de leur âge, de leur sexe et de leur place dans le réseau généalogique égocentré des systèmes dravidiens. Dès lors, les responsabilités et obligations rituelles, tout comme l'inceste, l'alliance et la descendance, se sont trouvées redéfinies à travers des catégories de parenté issues de l'appartenance à des sections et sous-sections, ne mobilisant plus la reconstitution dravidienne des liens généalogiques réels et classificatoires impliquant une forme d'interconnaissance. En d'autres termes, si le schème conceptuel sous-jacent à la mise en circulation des femmes (le don réciproque) n'a pas changé 
d'un système à l'autre, le problème posé par le brassage et la croissance démographique des groupes aborigènes a été résolu par l'activité métacognitive de leurs éminents représentants, qui a considérablement changé leurs principaux rapports sociaux. Par-delà le structuralisme, s'établit ainsi la convergence d'un certain matérialisme historique et naturalisme cognitif, si tant est que la politique et la pensée critique soient leur ruban de Möbius. 